\title{
The changing paradigm of ethics in uterus transplantation: a systematic review
}

Ledibabari M. Ngaage ${ }^{1}$, Samantha Ike ${ }^{2}$, Adekunle Elegbede ${ }^{3}$, Christian J. Vercler $^{4}$, Selim Gebran ${ }^{5}$, Fan Liang ${ }^{5}$, Erin M. Rada ${ }^{1}$, Carisa Cooney ${ }^{3}$, Gerald Brandacher ${ }^{3}$, Richard J. Redett ${ }^{3}$, Liza Johannesson ${ }^{6}$ \& Yvonne M. Rasko ${ }^{1}$ (D)

1 Division of Plastic Surgery, Department of Surgery, University of Maryland Medical Center, Baltimore, MD, USA

2 London North West University Healthcare Trust, London, UK

3 Department of Plastic \& Reconstructive Surgery, John Hopkins University School of Medicine, Baltimore, MD, USA

4 Plastic Surgery Section, Department of Surgery, University of Michigan, Ann Arbor, MI, USA

5 Division of Plastic \& Reconstructive Surgery, R Adams Cowley Shock Trauma Center, Baltimore, MD, USA

6 Annette C. and Harold C. Simmons Transplant Institute, Baylor University Medical Center, Dallas, TX, USA

\section{Correspondence}

Yvonne M. Rasko, Department of

Plastic Surgery, University of

Maryland, 22 S Greene St., Baltimore, MD 21230, USA.

Tel.: 410-328-2360;

fax: 410-328-0638;

e-mail: yrasko@som.umaryland.edu

\section{SUMIMARY}

The first uterus transplantation was performed in 2000. As key milestones are reached (long-lasting graft survival in 2011, and first birth from a transplanted womb in 2014), the ethical debate around uterus transplant evolves. We performed a systematic review of articles on uterus transplantation. Ethical themes were extracted and categorized according to four bioethical principles. Papers were divided into time periods separated by key events in uterus transplant history: Phase I (first technical achievement, 2002-2011), Phase II (clinical achievement, 2012-2014), and Phase III (after the first childbirth, 2015-2018). Eighty-one articles were included. The majority of ethics papers were published in Phase III $(65 \%$, $P<0.0001)$, that is after the first birth. Eighty percent of papers discussed nonmaleficence making it the most discussed principle. The first birth acted as a pivotal point: nonmaleficence was discussed by a lower proportion of articles $(P=0.0073)$, as was beneficence $(P=0.0309)$. However, discussion of justice increased to become the most discussed principle of the time period $(P=0.0085)$. The ethical debate surrounding uterus transplantation has evolved around landmark events that signify scientific progress. As safety and efficacy become evident, the focus of ethical debate shifts from clinical equipoise to socioeconomic challenges and equitable access to uterus transplantation.

Transplant International 2020; 33: 260-269

\section{Key words}

autonomy, beneficence, ethics, justice, nonmaleficence, uterus transplant

Received: 6 July 2019; Revision requested: 3 September 2019; Accepted: 28 October 2019; Published online: 6 January 2020

\section{Introduction}

The first human uterus transplantation (UTx) was performed in 2000 in Saudi Arabia from a living donor [1]. The graft underwent two cycles of withdrawal bleeding so was considered to be a technical achievement. However, the graft ultimately failed secondary to avascular necrosis three months later. The publication of this case sparked ethical discourse. The next reported advance in human uterus transplantation would not occur for another decade when a second uterus transplant was performed in Turkey in 2011; this time with a deceased donor [2]. Results illustrated proof of concept, as the graft demonstrated long-term survival and clinical pregnancy. However, true clinical success, defined as birth of viable offspring, was not achieved [3]. Subsequently, a clinical trial 
of uterus transplantation was performed in Sweden [4] which culminated in the first human birth in 2014 [5].

Since its inception, uterus transplantation has remained ethically controversial. The debate reflects the complexity and experimental nature of UTx including therapeutic misconception and its effect on informed consent [6]; the implications of use of living versus deceased donors $[7,8]$; and whether the risk-benefit ratio is justified [9]. These issues were partially addressed by the Montreal Criteria for ethical feasibility $[10,11]$. This guideline was established in 2012 and revised in 2013, when scientific success necessitated research and clinical communities to consider the ethical ramifications of UTx. It set the international standard for the ethical execution of uterine transplantation in humans. Later, UTx was encompassed under the umbrella of vascularized composite allotransplantation (VCAs) when the Organ Procurement and Transplant Network (OPTN) established a new regulatory guideline in the United States [12,13], which is used alongside the Montreal criteria.

Uterus transplantation, similar to other life-enhancing transplants, such as upper extremity and face, must achieve more than technical achievement as defined by mere allograft survival. Functional restoration (the ability to carry a pregnancy to term and give birth to a viable child) is the primary goal, without which, the clinical and scientific merit of UTx remains unestablished $[6,14]$. Some consider it to be a restorative surgery whose purpose is to treat infertility, and it is distinct because of its life-giving potential [15]. However, the potential benefits and risks to future offspring, donors and recipients must be considered. Deceased donor uteruses can be procured free from the physical risk posed to living donors but have had reduced success in achieving the primary goal [16]. The use of living donors in UTx is often compared with the use of gestational surrogates as both employ another's womb to achieve parenthood and involves risks to third parties $[10,11]$. Additionally, the recipient faces risk from immunosuppression; although ephemeral, this risk is combined with the need for multiple major surgeries (transplantation, caesarean section and graft explantation) [16]. These risks must then be weighed against potential benefits. Experience of childbearing and creation of a family are the primary motivations that drives the push for UTx. Body integrity, or the sense of 'feeling whole', can improve quality of life but is not considered of sufficient benefit to justify the substantial risks $[10,11,14]$. We must also consider challenges to informed consent and patient autonomy, such as therapeutic misconception. Therapeutic misconception occurs when patients fail to understand the difference between research and treatment which leads to unrealistic expectations [17]. Recipients must understand this to ensure valid consent and nonexploitation.

Currently, no literature documents the evolution of UTx ethics in response to scientific progress. It is important for clinicians to be aware of the ethical concerns surrounding this breakthrough treatment, and how literature addressing these concerns have evolved. Therefore, we performed a systematic review to evaluate how the ethical discourse surrounding uterus transplantation has evolved in relation to landmark events in the field.

\section{Materials and methods}

\section{Search strategy}

Following the Preferred Reporting Items for Systematic Reviews and Meta-Analyses guidelines, a literature search of four databases (PubMed, Embase, Cochrane and Scopus) was conducted from inception to 13 October 2018. The search strategy is available in Appendix S1. A manual search of the bibliographies of relevant papers was performed to identify additional studies for possible inclusion. Inclusion criteria consisted of articles that are (i) focused on human uterus transplantation and the ethical concerns related to it and (ii) written in the English language. Two independent reviewers (LMN and SI) performed the initial literature search and screening of titles and abstracts. This screened list of publications then underwent full article review by two independent reviewers (LMN and SI). Any disagreements regarding article inclusion were resolved by discussion.

\section{Thematic analysis}

We framed this review in the context of the four bioethical principles [18]: autonomy, beneficence, nonmaleficence and justice. Ethical themes were extracted qualitatively by two independent reviewers (LMN and SI) with the use of NVIVo (NVivo qualitative data analysis Software; QSR International Pty Ltd. Version 11, 2016, Melbourne, Australia) as an organizational tool to enable better tracking of thematic coding. Themes were then categorized according to Beauchamp and Childress' four principles: autonomy, beneficence, nonmaleficence and justice. Definitions of each of the four principles and how they relate to uterus transplantation are included in Table 1. 
Table 1. Descriptions of Beauchamp and Childress' four ethical principles and how each relates to uterus transplantation

\begin{tabular}{|c|c|c|}
\hline & Description & Relevance to UTx \\
\hline Autonomy & $\begin{array}{l}\text { Acknowledge and respect a } \\
\text { patient's right to choose free } \\
\text { from interference }\end{array}$ & $\begin{array}{l}\text { Recipients and donor must give voluntary informed consent without } \\
\text { pressure from external influences; recipients must be made aware } \\
\text { that UTx does not automatically equate to childbirth (therapeutic } \\
\text { misconception) }\end{array}$ \\
\hline Beneficence & $\begin{array}{l}\text { Always promoting good and } \\
\text { acting in the best interest of } \\
\text { the patient }\end{array}$ & $\begin{array}{l}\text { UTx has the potential to treat infertility, thus improving quality of life; } \\
\text { donors can receive psychological benefit through altruism; the } \\
\text { resultant functional gain can give life to children }\end{array}$ \\
\hline Nonmaleficence & $\begin{array}{l}\text { Primum non nocere - first, do } \\
\text { no harm. Either directly, } \\
\text { through adverse events or } \\
\text { absence of care }\end{array}$ & $\begin{array}{l}\text { Recipients undergo a minimum of three surgeries (transplantation, } \\
\text { Caesarean section and hysterectomy) and immunosuppression; } \\
\text { donors are at risk of exploitation, in addition to physical and } \\
\text { psychological harm; embryos face risk from immunosuppression in } \\
\text { utero }\end{array}$ \\
\hline Distributive justice & $\begin{array}{l}\text { Distributing potential benefits, } \\
\text { risks and costs fairly and } \\
\text { appropriately, treating all } \\
\text { patients in the same manner }\end{array}$ & $\begin{array}{l}\text { UTx is an alternative in cases where adoption/surrogacy is infeasible; } \\
\text { criteria must be set to prioritize recipient for uterus allocation; } \\
\text { funding is not yet in place so may only be financially feasible for the } \\
\text { affluent }\end{array}$ \\
\hline
\end{tabular}

UTx, uterus transplantation.

\section{Temporal analysis}

Medical advances are often provocative and ethical concerns can change in response to landmark events. This discourse can help or hinder research and thus influence future practice. Therefore, we analysed the change in ethics over time by subdividing the papers into groups according to key events in uterus transplantation: Phase I (following the first technical achievement, 2002-2011); Phase II (after proof of concept with the first clinical achievement, 20122014); and Phase III (following the first human birth from uterus transplantation, 2015-2018). Each subgroup begins the year after a landmark event to take into consideration the delay in time to publication.

\section{Data analysis}

Data were organized using Microsoft Excel (Microsoft 2016, Redmont, WA, USA) and analysed in SPSS (IBM Corp 2016. Armonk, NY, USA). Chi-square and Fisher's exact tests were used to compare percentages and proportions, as appropriate.
Figure 1 Flow chart demonstrating screening and selection of articles in literature search according to PRISMA guidelines.
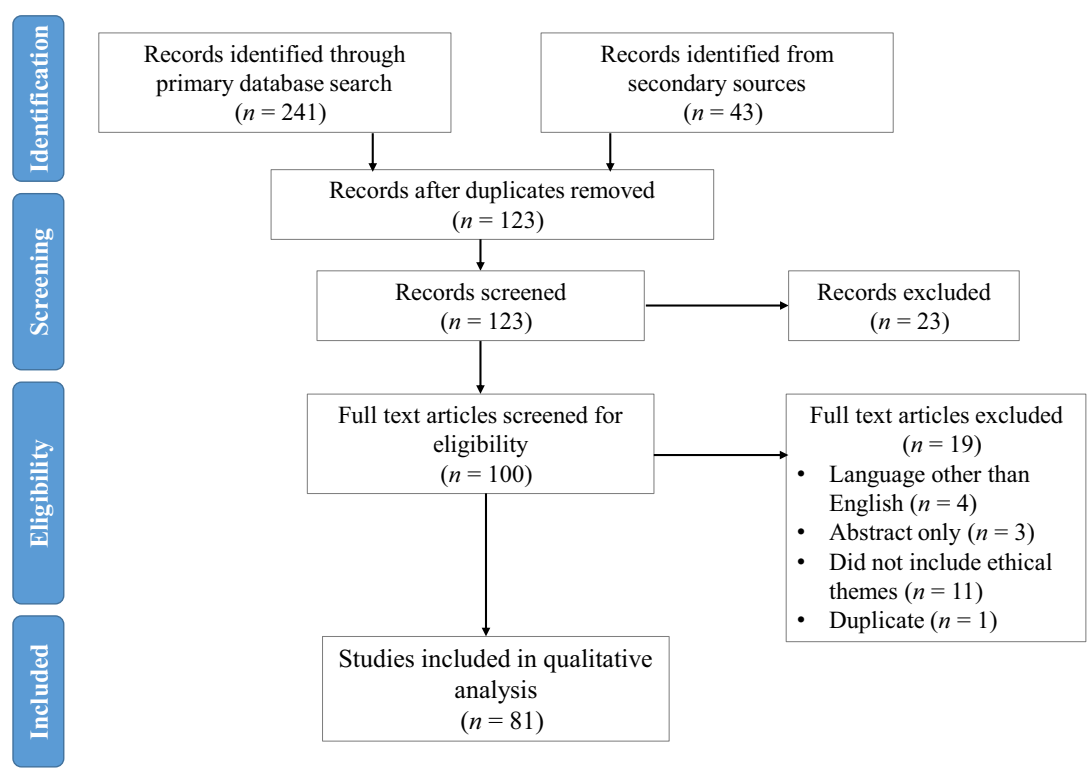
20

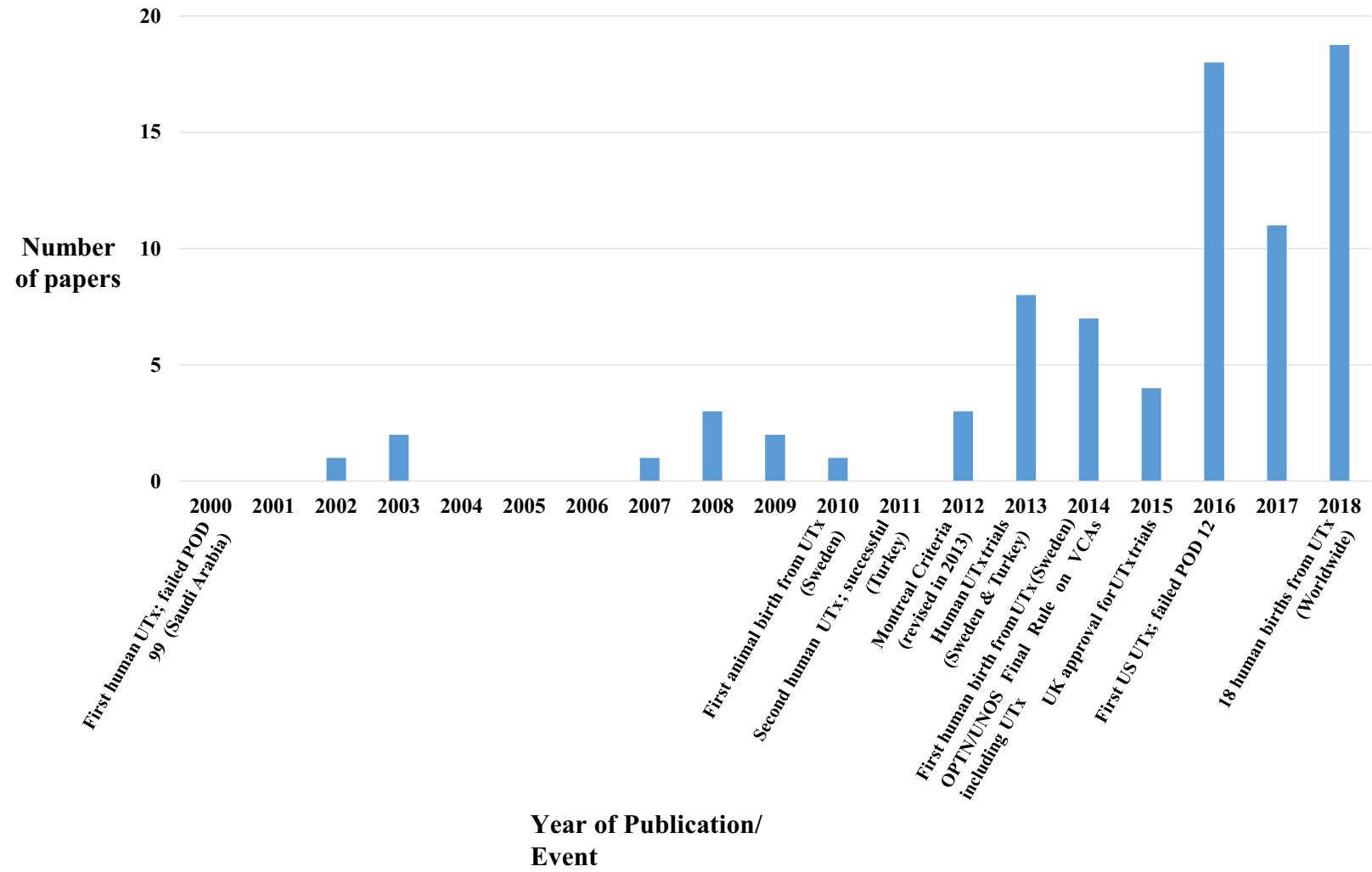

Figure 2 The number of publications on the ethics of uterus transplantation per year in relation to notable uterus transplant milestones. OPTN/UNOS, Organ Procurement and Transplantation Network/United Network for Organ Sharing; POD, postoperative day; UTx, uterus transplantation.

\section{Results}

We identified 284 citations. Following deduplication, we reviewed 123 unique articles. Of these, 81 articles met inclusion criteria and were included in the qualitative analysis (Appendix S2 and S3). Figure 1 summarizes the screening and article selection process.

Papers were published between 2002 and 2018. The mean number of publications per year was 4.8 [range: 0-20] (Fig. 2). Of the four bioethical principles, nonmaleficence was the most common theme (64 articles; $79 \%$ ), followed by justice (63 articles; 78\%) and autonomy (55 articles; 68\%), while beneficence (39 articles; $48 \%$ ) was least common.

\section{Subthemes}

Each bioethical principle encompasses a range of distinct subthemes, each with its own implications for surgical practice. Therefore, papers were further grouped by the primary subthemes within each principle.

Within the 64 articles on nonmaleficence, risk to living donor (surgical, psychological) $(n=39,61 \%)$ and the recipient (repeated surgeries, immunosuppression; $n=39,61 \%$ ) were the greatest concerns. This was followed by concerns on the immunosuppressive risk for the baby $(n=26,41 \%)$ and lack of regulation with risk of black market organ trade $(n=8,13 \%$; Fig. 3a).

Justice appeared in 63 publications, and its relevant subthemes included equity (equal opportunity for childbirth including alternative reproductive methods; $n=43,68 \%)$, allocation of transplant $(n=29,46 \%)$ and funding of the procedure ( $n=19,30 \%$; Fig. $3 \mathrm{~b}$ ).

Of the 55 papers featuring autonomy, they included subthemes of therapeutic misconception $(n=45,82 \%)$, informed consent of the donor $(n=27,49 \%)$ and selfdetermination of the recipient and donor (right to choose) ( $n=12,22 \%$; Fig. 3c).

Thirty-nine publications discussed beneficence with a primary focus on 'feeling whole' and improved quality of life of the recipient $(n=27,69 \%)$, reflecting the view of UTx as a life-enhancing procedure. Subthemes of functional restoration with childbirth $(n=22,56 \%)$ and the psychological benefit of donor altruism $(n=4$, $10 \%$ ) were also present (Fig. 3d). 
This data indicates that informed consent of recipient and donors (autonomy), improving quality of life (beneficence), mitigating risks to both recipients and donors (nonmaleficence) and ensuring reproductive equality (justice) are the most discussed ethical topics in uterus transplantation.

\section{Evolution of ethics}

There was an increase in publications after each phase with the majority of publications occurred after achievement of the primary goal with the first birth from a transplanted uterus (65\%). There was a significant increase in articles published between Phase III and Phases I $(P<0.0001)$, and II $(P<0.0001$; Fig. 4$)$, indicative of increasing interest in the ethics of uterus transplantation after achieving the primary goal of UTx. Within Phases II and III, there was a statistical difference between the proportions of at least two of the four bioethical principles discussed $(P=0.0442$ and $P<0.0001$, respectively; Table 2 ), signifying that the bioethical principles are not discussed equally in the UTx literature after the first clinical achievement in 2011.

When comparing the time periods after the first clinical achievement and first birth from a transplanted womb (Phases II versus III), we observed a significant decrease in the percentage of papers discussing nonmaleficence $(100 \%$ vs. $70 \%, \quad P=0.0073)$ and beneficence ( $72 \%$ vs. $42 \%, P=0.0309$ ). In addition, the proportion of papers discussing justice increased significantly from the first technical achievement to the first clinical achievement (Phase I: $40 \%$ vs. Phase II: $83 \%$, $P=0.0346)$ and after the first successful birth (Phase I: $40 \%$ vs. Phase III: $83 \%, P=0.0085$; Fig. 5). There was no difference between the proportions of papers discussing autonomy over time.

We then analysed subthemes for temporal changes. Within nonmaleficence, there was a notable decrease in the occurrence of 'immunosuppressive risk to newborn' after the first birth from a transplanted womb (Phase I: $67 \%$ vs. Phase II: $22 \%, P=0.0152)$. Conversely, there was an increase in concerns for the risks to living donors' at the same time point (Phase II: $39 \%$ vs. Phase III: $70 \%, P=0.0399$; Fig. 6). No significant temporal trends were noted in any subthemes within autonomy, beneficence or justice.

\section{Discussion}

With 18 human births from transplanted uteruses to date $[19,20]$, uterus transplantation is quickly becoming an established treatment alternative for women with absolute uterine infertility concerns. Advancements have been made at a rapid rate - progressing from the first human UTx failed attempt to a successful human birth in less than 15 years $[1,5]$. The ethical discourse has
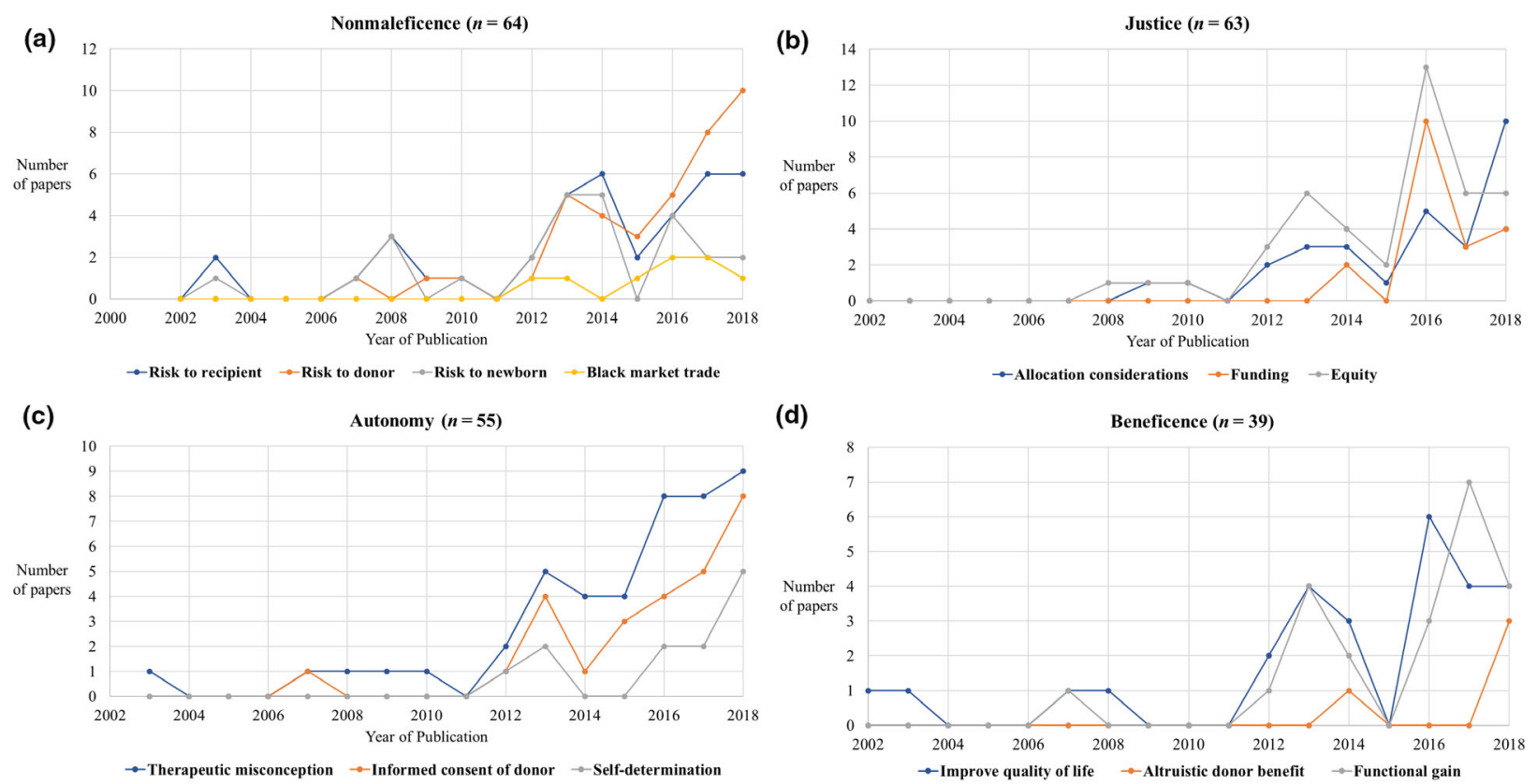

Figure 3 The major ethical subthemes of (a) nonmaleficence, (b) justice, (c) autonomy and (d) beneficence, and their appearance in literature from 2002 to 2018. 


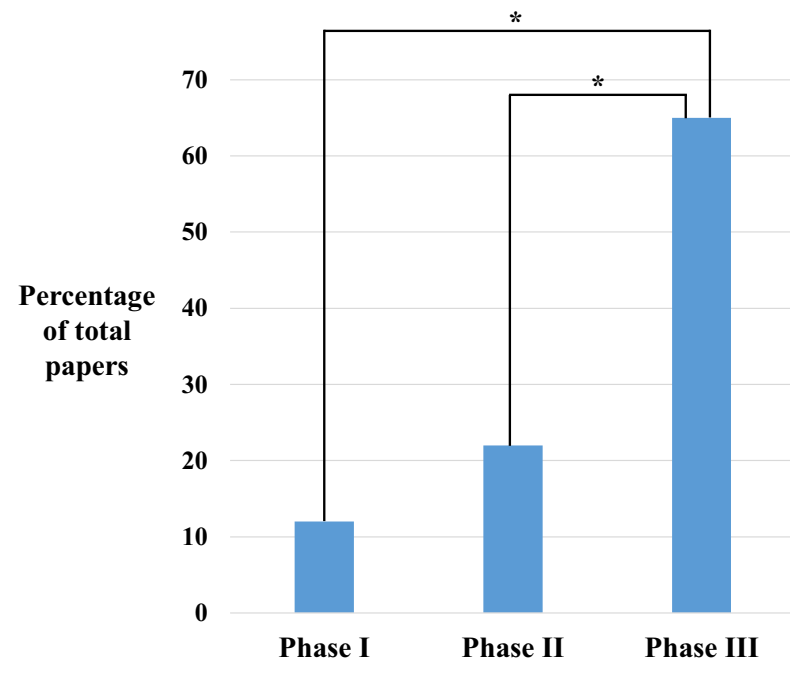

Figure 4 The number of ethics publications according to time period.

responded to and evolved with increasing clinical experience and successes. Furthermore, the unequivocal success of uterus transplant, demonstrated by the first viable human birth in 2014, acts as a pivotal point.

The ethical prerequisite for non-life-saving transplants relies on the prospect of either decreased morbidity or superior outcomes, when compared with the alternatives. Morbidity from uterine transplantation includes effects of immunosuppression, and the risk of surgery to both donor and recipient. Success of UTx is determined by a clear endpoint: childbirth. We are in strong support of uterus transplantation when ethical benchmarks are met. Based on the results of our study and previous ethical guidelines [10,11,21], we summarized ethical considerations and recommendations for uterus transplantation (Table 3).

The progression of ethical discourse follows a pattern seen in other VCA transplants [22,23]. The first stage coincides with initial scientific attempts when we ask 'what can we do?' As advances occur, the question shifts to 'what should we do?' This is demonstrated by the increase in publications and the establishment of a regulatory ethics framework after the first clinical achievement in 2011 [10]. This question gains more importance when the procedure is not life-saving but life-enhancing, such

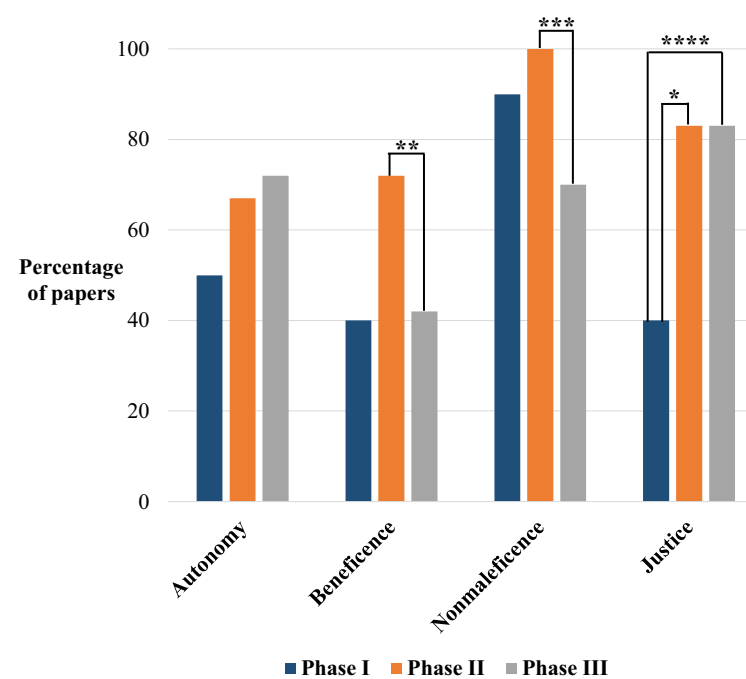

Figure $\mathbf{5}$ The percentage of papers discussing autonomy, beneficence, nonmaleficence and justice divided by time period. ${ }^{\star} P=0.0346,{ }^{*} * P=0.0309,{ }^{*} * * P=0.0073, * * * * P=0.0085$.

as face and upper extremity transplantation, which have, and continue to face, similar ethical challenges as they become more successful [24,25]. During these early stages, the principle of nonmaleficence dominates, a trend that is also reflected in the upper extremity and face transplantation literature $[22,23]$. This may not be surprising given the physician's doctrine of 'do no harm'. Not only is there clinician hesitation to place patients at risk through experimental therapies, but there are also systematic procedures and checks to identify, mitigate and remove risk to patients. Ethics literature is centred on proving the procedure has low enough risk relative to any potential benefit. This may be reflected in our results; wherein beneficence is the principle that is least discussed in the UTx literature. Alternatively, in the case of UTx, where the beneficence is more clearly apparent, it may be that the potential benefits require less discussion.

There was also an emphasis on ensuring informed consent from the recipient as evidenced by the prevalence of the 'therapeutic misconception' subtheme. Classically, therapeutic misconception is defined as 'when individuals do not understand that the defining purpose of clinical research is to produce generalizable knowledge, regardless

Table 2. Proportion of papers within each time period that discussed autonomy, beneficence, nonmaleficence or justice

\begin{tabular}{lllllr}
\hline & Autonomy (\%) & Beneficence (\%) & Nonmaleficence (\%) & Justice (\%) & $P$-value \\
\hline Phase I $(n=10)$ & 50 & 40 & 90 & 40 & 0.0749 \\
Phase II $(n=18)$ & 67 & 72 & 100 & 83 & 0.0442 \\
Phase III $(n=53)$ & 72 & 42 & 70 & 83 & $<0.0001$ \\
\hline
\end{tabular}


(a)

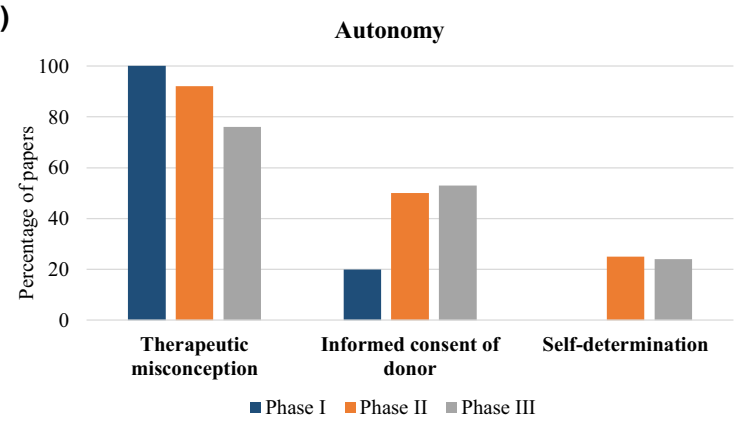

(c)

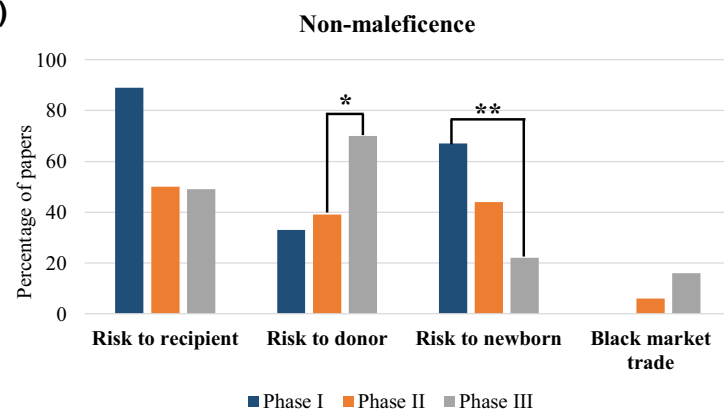

(b)

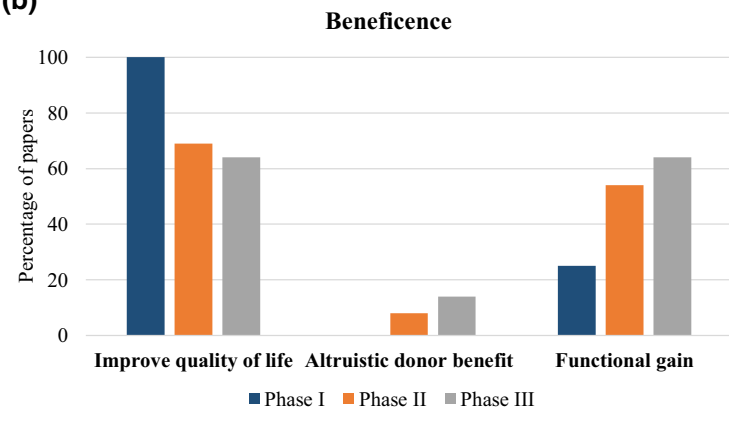

(d)

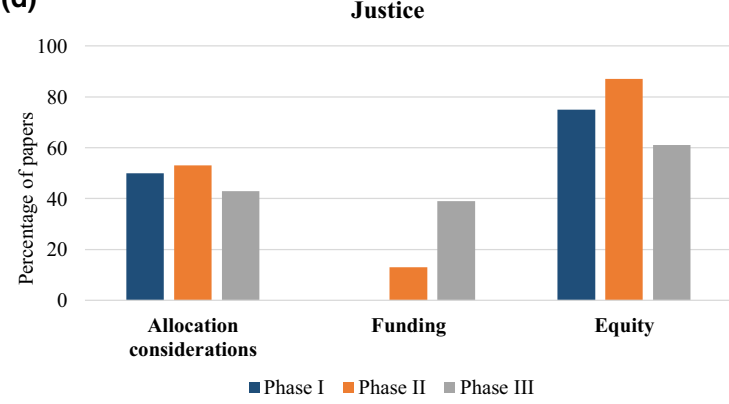

Figure 6 The percentage of papers discussing each subtheme of the four bioethical principles: (a) autonomy, (b) beneficence, (c) nonmaleficence and (d) justice, according to time period. ${ }^{*} P=0.0399,{ }^{*} P=0.0152$.

Table 3. Ethical considerations for uterus transplantation $[10,11,16,21]$

\begin{tabular}{ll}
\hline $\begin{array}{l}\text { Aspect of uterus } \\
\text { transplantation }\end{array}$ & $\begin{array}{c}\text { Recommendation to ensure ethical } \\
\text { conduction of uterus transplantation }\end{array}$ \\
\hline Indication & $\begin{array}{c}\text { Experience of pregnancy and birth of } \\
\text { viable child } \\
\text { Candidate }\end{array}$ \\
& $\begin{array}{c}\text { Physically healthy and able to withstand } \\
\text { immunosuppression } \\
\text { Passes psychological assessment } \\
\text { Possesses viable embryos available for } \\
\text { implantation } \\
\text { Understands the temporary nature of } \\
\text { uterus transplant } \\
\text { Deceased donors: Loved ones must give } \\
\text { informed consent } \\
\text { Living donors: Understands risks of } \\
\text { hysterectomy and gives informed } \\
\text { consent } \\
\text { Foetal preterm and post-term } \\
\text { monitoring }\end{array}$ \\
\hline
\end{tabular}

of whether the subjects enrolled in the trial may potentially benefit from the intervention' [26]; and it is often seen in discussion of experimental therapies. In the context of UTx, it relates to patients' unrealistic expectations that may prevent true informed consent. Thus, the prevalence of this subtheme is consistent with the status of
UTx as an experimental therapy rather than the standard of care. As the uncertainty of outcomes decreases with each new birth, UTx will likely transition in status, marking another milestone.

The first successful human birth from a transplanted womb in 2014 was a watershed moment for the ethical discourse surrounding uterus transplantation. We see a relative decrease in discourse around principles of nonmaleficence and beneficence whereas the discussion of justice more than doubles to become the most prevalent theme within the same time period. It is clear that as the questions of safety and efficacy are answered, a new question arises: how can we do this fairly? Concerns about use of alternative fertility methods and ensuring equity in reproduction dominated the discussion, quickly followed by organ allocation and financial concerns. This progression from ethical questioning to addressing socioeconomic equity issues is mirrored in other organ transplantations [22,23,27].

Uterus transplantation is seen as an alternative in cases where surrogacy is illegal and a genetic connection to the child is sought, thereby increasing a woman's reproductive autonomy [28]. The recommendations emphasize that experience of childbearing and creation of a family should be the primary motivation. Interestingly, unlike other life-enhancing transplantations, body integrity is not deemed a sufficient indication for UTx. This is a 
point of contention as the debate moves to inclusion of transgender women [29]. Established recommendations on how to prioritize recipients for this scarce resource are necessary to ensure equitable distribution and access to treatment $[10,11]$. The need to impose structure and regulations in allocation is highlighted in the corresponding increase in the appearance of the subtheme of black market trade (nonmaleficence) in the literature. In practice, lack of regulation can become synonymous with permission [29], and similar issues have been observed in renal transplantations in which organs can be sourced from living donors, in addition to the deceased [30]. Some countries have imposed strict bans, while others have permitted commercial sale [31,32]. The debate on organ transplant tourism is still ongoing $[33,34]$. This may be further exacerbated by the uncertainty in funding sources. Although the surgical procedure could be covered once the patient is approved for treatment, the ability to obtain a viable embryo is crucial in order to give birth through UTx. Therefore, access to in vitro fertilization (IVF) is often required to be eligible for UTx [21]. This may limit access to UTx to those who can afford this additional procedure.

The impact of the first birth from UTx was also seen within the subthemes, most notably for those within the nonmaleficence principle. The overall downtrend in the discussion of nonmaleficence may be attributed to the 'risk to recipient' and 'immunosuppressive risk to the newborn' subthemes. In reconstructive transplantation, potential harm to the recipient primarily relate to the requirement of long-term immunosuppression that can result in reduced life expectancy [35]. However, unlike upper extremity and face transplantation, UTx is intended to be temporary, in that the graft is removed following childbirth, as the last of a series of major operations. As such, immunosuppression is not lifelong, which adds a dimension that does not exist for other life-enhancing transplants. It would therefore be reasonable to expect that over time, as more UTx recipients undergo graft explantation following childbirth, concerns regarding long-term toxicity of immunosuppression may further decrease. To adequately prepare the patient prior to transplantation, counselling should emphasize plans for explantation after childbirth, as well as explantation in the case of failure to conceive or deliver a viable child with the graft. These discussions should be ongoing throughout the course of treatment. At this time, no evidence-based recommendations for the maximum duration to retain a transplanted uterus have been established. We believe that these decisions should be individualized, based on the patient's clinical status, goals and continuing discussion of risks and benefits between the patient and transplant team.

Clinical evidence may also assuage ethical concerns for immunosuppressive risk to the newborn. This is illustrated by the decrease of this subtheme in the literature after the first viable birth. Additionally, there is wealth of information from solid organ transplantation demonstrating the safety of pregnancies in patients on immunosuppression [36]. Approximately 14\% of recipients of nonuterus, solid organ transplants are women in their childbearing years, and pregnancy after transplantation is becoming increasingly common [37]. Women who become pregnant after solid organ transplant face greater risks when pregnant compared with UTx recipients. They have often received immunosuppression for significantly longer, have comorbidities and chronic disease, such as hypertension and diabetes, which add additional risks to pregnancy, and are unable to stop immunosuppression during pregnancy or risk losing the transplanted organ. Strategies are in place to ensure safe and successful birth in a post-transplant setting (Table 4).

Strikingly, there was a notable increase in concerns for living donors in the same time period as the downtrend of the nonmaleficence theme. In contrast to other VCA transplants, the UTx donor pool includes living donors. In VCA transplants, such as face, penis and upper extremity, donor grafts are limited to deceased donors. To date, most births from UTx have resulted from living uterus donation, and increased success results in increased demand. Thus, there is increased interest in use of living donors. The inclusion of living donors in UTx means that donor ethics more closely resemble that of other living solid organ transplants, such as kidney or liver, than other VCA transplants. Living donor transplantation is controversial because it exposes donors to risks for the potential benefit of third parties (recipients) [38]. The potential risks must be balanced with the donor's autonomy and right to choose. The risk of

Table 4. Strategies for successful pregnancy after solid organ transplantation [37]

1. Accurate and early diagnosis and dating of pregnancy

2. Close monitoring of graft function and immunosuppressive drug levels

3. Maternal surveillance for hypertension, gestational diabetes, pre-eclampsia and bacterial or viral infection

4. Foetal preterm surveillance for malformation, foetal growth and well-being

5. Aim to deliver at term 
hysterectomy in this population is unknown, although serious complications including reoperation have been reported [39]. However, uteruses are sourced from donors who no longer wish to childbear and, therefore, have exhausted their function. Nevertheless, living donations are fraught with the potential for commercial sale of organs [40-42]. This concern is highlighted by the appearance of the subtheme of black market trade (nonmaleficence) in our results. In practice, lack of regulation can become synonymous with permission [43], and similar issues have been observed in renal transplantations in which organs can be sourced from living donors, in addition to the deceased [43]. Therefore, established recommendations on how to prioritize recipients for this scarce resource are necessary to ensure equitable distribution and access to treatment. Recent births have occurred from deceased donor uteruses $[19,20]$, so it is also possible that this UTx subtheme will trend down in the future.

There are several limitations within this review. Although an exhaustive literature search was conducted, it is possible that relevant articles were excluded. In addition, we did not include media and news articles. Although the majority of publications explicitly named at least one of the bioethical principles within the text, several articles were less direct in stating the primary bioethical principles of interest. Therefore, some interpretation by the authors was required to identify the primary themes. To minimize the procrustean nature of this approach, subthemes were extracted first and after discussion were arranged into the bioethical principles. Additionally, two independent reviewers analysed each article to reduce the effect of reviewer subjectivity. Another limitation is the different group sizes of publications in all three phases, as well as the low number of publications in the first era. Therefore, caution should be taken when interpreting results.

We have highlighted the past and current discussion on the ethics of uterus transplantation in the hope of advancing the debate. The evolution of ethics in uterus transplantation follows a pattern that has been previously seen in other life-enhancing transplants: initial clinical hesitancy as we venture into the unknown, followed by an emphasis on prevention of harm and ensuring informed consent, and with increasing acceptance comes a focus on equitable access and socioeconomic challenges. These changes are in response to landmark events in UTx that signify scientific progress in the area, and the first birth from a transplanted womb, the measurement of true success in a uterus transplant, acts as a pivotal point in the discussion.

\section{Authorship}

LMN: designed study, collected data, analysed data, initial manuscript, manuscript editing and final manuscript approval. SI: collected data, analysed data, manuscript editing and final manuscript approval. AE: designed study, analysed data, manuscript editing and final manuscript approval. CJV: designed study, manuscript editing and final manuscript. SG, MD: designed study, manuscript editing and final manuscript. FL: designed study, manuscript editing and final manuscript. EMR: designed study, manuscript editing and final manuscript. CC: designed study, analysed data, manuscript editing and final manuscript. GB: analysed data, manuscript editing and final manuscript. RJR: analysed data, manuscript editing and final manuscript. LJ: designed study, analysed data, manuscript editing and final manuscript. YMR: designed study, analysed data, manuscript editing and final manuscript approval.

\section{Funding}

The authors have declared no funding.

\section{Conflicts of interest}

The authors have declared no conflicts of interest.

\section{Acknowledgement}

None.

\section{SUPPORTING INFORMATION}

Additional supporting information may be found online in the Supporting Information section at the end of the article.

Appendix S1. Contains the search strategy for the systematic review.

Appendix S2. Contains the results of literature search and the relevant bioethical principles associated with each publication.

Appendix S3. Contains the references for articles found in literature search and listed in Appendix S2. 
1. Fageeh W, Raffa H, Jabbad H, et al. Transplantation of the human uterus. Intl J Gynaecol Obstet 2002; 76: 145.

2. Ozkan O, Akar ME, Ozkan O. Preliminary results of the first human uterus transplantation from a multiorgan donor. Fertil Steril 2013; 99: 470.

3. Erman Akar M, Ozkan O, Aydinuraz B, et al. Clinical pregnancy after uterus transplantation. Fertil Steril 2013; 100: 1358.

4. Brannström M, Johannesson L, DahmKahler $\mathrm{P}$, et al. First clinical uterus transplantation trial: a six-month report. Fertil Steril 2014; 101: 1228.

5. Brannström M, Johannesson L, Bokström $\mathrm{H}$, et al. Livebirth after uterus transplantation. Lancet 2015; 385: 607.

6. Caplan AL, Perry C, Plante LA, et al. Moving the womb. Hastings Cent Rep 2007; 37: 18.

7. Dickens BM. Legal and ethical issues of uterus transplantation. Int $J$ Gynaecol Obstet 2016; 133: 125.

8. Olausson M, Johannesson L, Brattgård $\mathrm{D}$, et al. Ethics of uterus transplantation with live donors. Fertil Steril 2014; 102: 40.

9. Johannesson L, Dahm-Kähler P, Eklind $\mathrm{S}$, et al. The future of human uterus transplantation. Womens Health (Lond) 2014; 10: 455.

10. Lefkowitz A, Edwards M, Balayla J. The Montreal criteria for the ethical feasibility of uterine transplantation. Transpl Int 2012; 25: 439.

11. Lefkowitz A, Edwards M, Balayla J. Ethical considerations in the era of the uterine transplant: an update of the Montreal Criteria for the Ethical Feasibility of Uterine Transplantation. Fertil Steril 2013; 100: 924.

12. OPTN. The Status of Vascularized Composite Allograft Allocation, 2014. Available from: http://optn.transplant. hrsa.gov/news/the-statusof-vascula rized-composite-allograft-allocation/. Accessed 5th November 2018.

13. Glazier AK. Regulatory oversight in the United States of vascularized composite allografts. Transplant Int 2016; 29: 682.

14. Allyse M. "Whole again": why are penile transplants less controversial than uterine? Am J Bioeth 2018; 18: 34.

15. Johannesson L, Järvholm S. Uterus transplantation: current progress and future prospects. Int $J$ Womens Health 2016; 8: 43.
16. Bruno B, Arora KS. Uterus transplantation: the ethics of using deceased versus living donors. Am J Bioeth 2018; 18: 6.

17. Catsanos R, Rogers W, Lotz M. The ethics of uterus transplantation. Bioethics 2013; 27: 65.

18. Beauchamp T, Childress J. Principles of Biomedical Ethics, 18th ed. New York, NY: Oxford University Press, 2009.

19. Diaz-Garcia C, Pellicer A. Uterus transplantation from a deceased donor. Lancet 2018; 392: 2657.

20. Ejzenberg D, Andraus W, Baratelli Carelli Mendes LR, et al. Livebirth after uterus transplantation from a deceased donor in a recipient with uterine infertility. Lancet 2018; 392: 2697.

21. Bayefsky M, Berkman B. Toward the ethical allocation of uterine transplants. Am J Bioeth 2018; 18: 16.

22. Cooney CM, Siotos C, Aston JW, et al. The ethics of hand transplantation: a systematic review. J Hand Surg Am 2018; 43: 84.el.

23. Kiwanuka H, Bueno EM, Diaz-Siso JR, et al. Evolution of ethical debate on face transplantation. Plast Reconstr Surg 2013; 132: 1558.

24. Theodorakopoulou E, Meghji S, Pafitanis G, et al. A review of the world's published face transplant cases: ethical perspectives. Scars Burn Heal 2017; 3: 2059513117694402.

25. Breidenbach WC, Meister EA, Turker $\mathrm{T}$, et al. A methodology for determining standard of care status for a new surgical procedure: hand transplantation. Plast Reconstr Surg 2016; 137: 367.

26. Henderson GE, Churchill LR, Davis $\mathrm{AM}$, et al. Clinical trials and medical care: defining the therapeutic misconception. PLoS Med Medicine 2007; 4: e324.

27. Nadiminti H. Organ transplantation: a dream of the past, a reality of the present, an ethical challenge for the future. Virtual Mentor 2005; 7: 32.

28. Robertson JA. Other women's wombs: uterus transplants and gestational surrogacy. J Law Biosci 2016; 3: 68.

29. Spillman M, Sade R. A woman in full. Am J Bioeth 2018; 18: 32.

30. Surman OS, Saidi R, Burke TF. Regulating the sale of human organs: a discussion in context with the global market. Curr Opin Organ Transplant 2008; 13: 196.
31. Rizvi AH, Naqvi AS, Zafar NM, et al. Regulated compensated donation in Pakistan and Iran. Curr Opin Organ Transplant 2009; 14: 124.

32. Cohen IG. Can the government ban organ sale? Recent court challenges and the future of US law on selling human organs and other tissue. Am J Transplant 2012; 12: 1983.

33. Ghods AJ. Changing ethics in renal transplantation: presentation of Iran model. Transplant Proc 2004; 36: 11.

34. Piccoli GB, Sacchetti L, Verzè L, et al. Doctor can I buy a new kidney? I've heard it isn't forbidden: what is the role of the nephrologist when dealing with a patient who wants to buy a kidney? Philos Ethics Humanit Med 2015; 10: 13.

35. Hsu DC, Katelaris CH. Long-term management of patients taking immunosuppressive drugs. Aust Prescr 2009; 32: 68 .

36. Durst JK, Rampersad RM. Pregnancy in women with solid-organ transplants: a review. Obstet Gynecol Surv 2015; 70: 408.

37. Deshpande NA, Coscia LA, Gomez-Lobo V, Moritz MJ, Armenti VT. Pregnancy after solid organ transplantation: a guide for obstetric management. Rev Obstet Gynecol. 2013; 6: 116.

38. Wright L, Faith K, Richardson R, Grant D, Joint Centre for Bioethics, University of Toronto, Toronto, Ont. Ethical guidelines for the evaluation of living organ donors. Can J Surg 2004; 47: 408.

39. Jones BP, Saso S, Bracewell-Milnes T, et al. Human uterine transplantation: a review of outcomes from the first 45 cases. BJOG 2019; 126: 1310.

40. Colakoglu M, Yenicesu M, Akpolat T, et al. Nonrelated living-donor kidney transplantation: medical and ethical aspects. Nephron 1998; 79: 447.

41. Cohen IG. Can the government ban organ sale? Recent court challenges and the future of US law on selling human organs and other tissue. Am J Transplant. 2012; 12: 1983.

42. Rizvi AH, Naqvi AS, Zafar NM, et al. Regulated compensated donation in Pakistan and Iran. Curr Opin Organ Transplant. 2009; 14: 124.

43. Surman OS, Saidi R, Burke TF. Regulating the saleof human organs: a discussion in context with the global market. Curr Opin Organ Transplant. 2008; 13: 196. 